\title{
On decay centrality in graphs
}

\author{
J. Coroničová Hurajová ${ }^{a}$, S. Gago ${ }^{b *}$ and T. Madaras ${ }^{c}$
}

June 2, 2015

${ }^{a}$ The Faculty of Business Economics with seat in Košice, The University of Economics in Bratislava, Tajovského 13, 04130 Košice, Slovakia email: jana.coronicova.hurajova@euke.sk

${ }^{b}$ Dept. Matemàtica Aplicada III, EUETIB, Universitat Politècnica de Catalunya Comte d'Urgell 187, 08036 Barcelona, Spain email: silvia.gago@upc.edu

${ }^{c}$ Institute of Mathematics, Jesenná 5, 04001 Košice, Slovakia email: tomas.madaras@upjs.sk

\begin{abstract}
The decay centrality of a vertex $v$ of a graph $G$ is defined as the value $\sum_{u \in V(G)} \delta^{d(u, v)}$ where $d(u, v)$ is the shortest path distance and $\delta \in(0,1)$ is a parameter. This invariant (introduced independently by Jackson and Wolinsky,1996 and Dangalchev, 2011) is considered as a replacement for the closeness centrality for disconnected graphs, however, the objection on its inconsistency with the closeness was pointed out by Yang and Zhuhadar (2011). We explore mathematical properties of decay centrality depending on the choice of parameter $\delta$, and the stability of vertex ranking based on this centrality index.
\end{abstract}

Keywords: decay centrality, decay-stable graphs

\section{Introduction}

Throughout this paper, we consider graphs without loops or multiple edges; we use the standard graph terminology as used in Diestel (2005). Given a graph $G$, the symbols $V(G), E(G)$ stand for the vertex set and the edge set of $G$,

${ }^{*}$ Research supported by the Ministry of Science and Technology (Spain) under project MTM2010-19660 
respectively. The distance $d(x, y)$ of vertices $x, y \in V(G)$ is the length of a shortest $x-y$-path (if no $x-y$-path exists in $G$, we set $d(x, y)=+\infty$ ); the maximum distance of vertices of $G$ is called the diameter $\operatorname{diam}(G)$ of $G$.

Among fundamental questions discussed in the analysis of social networks of relations between actors, an important task is to identify the actors which play, within a network, a key role. The usual way to express a measure of their importance involves the centrality index, formally defined, for a graph $G$, as a function $c: V(G) \rightarrow \mathbb{R}$ which is invariant under graph isomorphism (for interpretation purposes, the vertices of $G$ with higher centrality values correspond to more important actors of the network modelled by $G$ ). The most frequently used centrality indices are vertex degree, eccentricity, closeness and betweenness; for the detailed discussion on their properties and usage, see Chapters 3, 4, and 5 in Brandes and Erlebach (2005).

In search for another approaches in the area of centrality research, several new centrality indices were defined. One of them is reciprocal distances centrality (see Latora and Marchiori 2001; Knor and Madaras 2004) defined as

$$
C_{R}(x)=\sum_{y \in V(G) \backslash\{x\}} \frac{1}{d(x, y)}
$$

and another one is the decay centrality defined in Jackson et al. (2008) (see also Jackson and Wolinsky 1996) and independently in Dangalchev (2011) (under the name generalized closeness) as

$$
C_{\delta}(x)=\sum_{y \in V(G) \backslash\{x\}} \delta^{d(x, y)}
$$

where $\delta \in(0,1)$ is a parameter (usually equal to $\frac{1}{2}$, see the Section 5 of Dangalchev 2011 for a discussion on the choice of $\delta$ ). These indices overcome the known deficiency of the closeness: the zero value for all vertices of disconnected graphs. On the other hand, Yang and Zhuhadar (2011) argue that $C_{R}$ and $C_{\delta}$ cannot be considered as extension of the closeness for disconnected graphs, because, for connected graphs, they lead to rankings of vertices which are not mutually consistent - they show that, for the complete binary tree $T$ of height two, the closeness centrality ranks its root (that is, the vertex of degree 2 in $T$ ) as the unique most central vertex while the reciprocal distance centrality ranks, as the most central vertices, two vertices of $T$ that have degree 3. Furthermore, using the generalized closeness for $\delta=\frac{1}{2}$, one obtains that all three non-pendant vertices of $T$ are the most central.

Rather than advocating either opinion, we present an opinion that the ranking order anomaly reported in Yang and Zhuhadar (2011) is a new kind of phenomenon intrinsically connected with properties of decay centrality. In order to formally describe this phenomenon, define, for a graph $G$ with $V(G)=$ $\left\{v_{1}, \ldots, v_{n}\right\}$ and a general centrality index $c: V(G) \rightarrow \mathbb{R}$, the $c$-ranking of $G$ in the following way: let $\pi$ a the permutation of $\{1, \ldots, n\}$ such that $\left(c\left(v_{\pi(1)}\right), \ldots, c\left(v_{\pi(n)}\right)\right)$ is a non-increasing sequence (in other words, it sorts the 
vertices starting from the highest centrality) with $\ell$ blocks $\left(c\left(v_{\pi(1)}\right), \ldots, c\left(v_{\pi\left(k_{1}\right)}\right)\right)$, $\left(c\left(v_{\pi\left(k_{1}+1\right)}\right), \ldots, c\left(v_{\pi\left(k_{2}\right)}\right)\right), \ldots,\left(c\left(v_{\pi\left(k_{\ell-1}+1\right)}\right), \ldots, c\left(v_{\pi(n)}\right)\right)$; in this sequence, distinct blocks contain distinct values whereas the values within a block are the same. Then the $c$-ranking of $G$ is the sequence $\left(\overline{v_{\pi(1)}, \ldots, v_{\pi\left(k_{1}\right)}}, \overline{v_{\pi\left(k_{1}+1\right)}, \ldots, v_{\pi\left(k_{2}\right)}}\right.$, $\ldots, \overline{v_{\pi\left(k_{\ell-1}+1\right)}, \ldots, v_{\pi(n)}}$ ) (the lines over vertices indicate the fact that centralities of vertices within the same group are equal). In the case of $c=C_{\delta}$, the $c$-ranking of a graph depends on $\delta$; taking $\left(\begin{array}{c}n \\ 2\end{array}\right)$ polynomials $C_{\delta}\left(v_{i}\right)-C_{\delta}\left(v_{j}\right)$ for all $i, j \in\{1, \ldots, n\}$, their roots in $(0,1)$ are called decay thresholds of $G$.

Now, if we denote the root of the above mentioned tree $T$ as $u_{1}$, its neighbours as $u_{2}, u_{5}$ and the neighbours of $u_{2}$ and $u_{5}$ as $u_{3}, u_{4}$ and $u_{6}, u_{7}$, respectively, we can observe that $T$ has the unique decay threshold at $\frac{1}{2}$ and $C_{\delta}$-ranking of $T$ is $\left(\overline{u_{2}, u_{5}}, u_{1}, \overline{u_{3}, u_{4}, u_{6}, u_{7}}\right)$ for $0 \leq \delta<\frac{1}{2},\left(\overline{u_{1}, u_{2}, u_{5}}, \overline{u_{3}, u_{4}, u_{6}, u_{7}}\right)$ for $\delta=\frac{1}{2}$ and $\left(u_{1}, \overline{u_{2}, u_{5}}, \overline{u_{3}, u_{4}, u_{6}, u_{7}}\right)$ for $\frac{1}{2}<\delta<1$ (see Figure 1 for graphs of $C_{\delta}$ ).

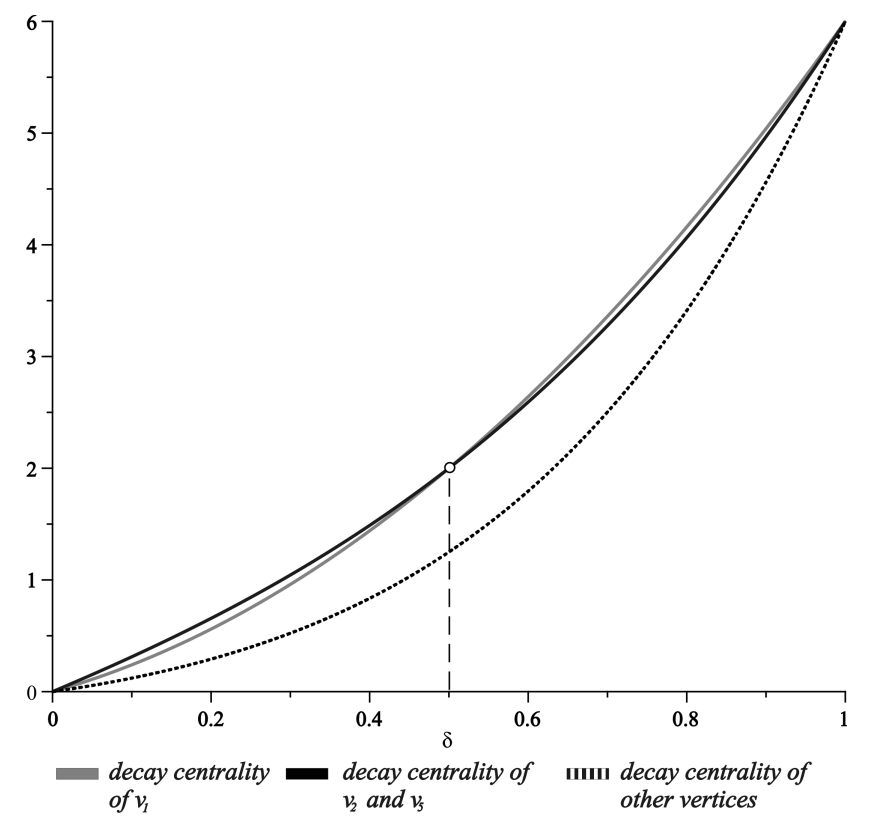

Figure 1: Graphs of decay centralities for the complete binary tree of height 2

Hence, $C_{\delta}$-ranking may vary, in general, with different values of $\delta$; its behaviour and general properties are explored in a detail in Section 2.

On the other hand, there are graphs for which $C_{\delta}$-ranking does not change; we will call these graphs as decay-stable and discuss their properties in Section 3. We show that the decay ranking of vertices is preserved within graphs of various graph products, and, also, within graphs of diameter two. However, we also exhibit several real-world networks whose decay rankings are highly unstable. These findings suggest that the decay centrality, although having some advantages over the closeness centrality, is perhaps not a good choice for analyzing real data. 


\section{General properties}

The decay centrality of a vertex $v$ of a graph $G$ is a polynomial of degree $k=e(v)$, the eccentricity of $v$ (that is, the maximum of distances of vertices from $v)$; its coefficients are equal to terms of the distance degree sequence $D D S(v)$ of $v$ which is the sequence $\left(1, d_{1}, \ldots, d_{k}\right)$ where $d_{i}$ is the number of vertices of the distance $i$ from $v$ (see Bloom et al. 1983). It is easy to see that, in graphs whose vertices have the same distance degree sequences (distance degree regular graphs or DDR graphs for short), all vertices have the same decay centrality. Conversely, if $G$ is a graph such that, for any pair $x, y$ of its vertices, $C_{\delta}(x)=$ $C_{\delta}(y)$ for all $\delta \in(0,1)$, then $G$ is a DDR graph. Note also that the vertices of each DDR graph have the same closeness; the converse, however, is not true, as seen on the graph $C_{12}^{\times}$constructed from a 12-cycle $v_{1} v_{2} \ldots v_{12}$ by adding new edges $v_{1} v_{3}, v_{2} v_{4}, v_{5} v_{7}, v_{6} v_{8}, v_{9} v_{11}, v_{10} v_{12}$ : its vertices have two different DDS sequences (hence, two different decay centralities), but the same closeness.

Proposition 1. An universal vertex of a graph has, for any $\delta \in(0,1)$, the maximum decay centrality among all vertices.

Lemma 1. Let $G$ be a graph and $x, y$ be its vertices such that $e(x)=2, e(y) \geq 3$ and $\operatorname{deg}(x) \geq \operatorname{deg}(y)$. Then, for all $\delta \in(0,1), C_{\delta}(x)>C_{\delta}(y)$.

Proof. If $D D S(x)=\left(1, x_{1}, x_{2}\right)$ and $D D S(y)=\left(1, y_{1}, \ldots, y_{k}\right), k=e(y)$, then $C_{\delta}(x)-C_{\delta}(y)=\left(x_{1}-y_{1}\right) \delta+\left(x_{2}-y_{2}\right) \delta^{2}-\sum_{i=3}^{k} y_{i} \delta^{i} \geq\left(x_{1}-y_{1}\right) \delta^{2}+\left(x_{2}-y_{2}\right) \delta^{2}-$ $\sum_{i=3}^{k} y_{i} \delta^{i}=\left(x_{1}+x_{2}-y_{1}-y_{2}\right) \delta^{2}-\sum_{i=3}^{k} y_{i} \delta^{i}=\delta^{2} \sum_{i=3}^{k} y_{i}-\sum_{i=3}^{k} y_{i} \delta^{i}=\delta^{2} \sum_{i=3}^{k} y_{i}(1-$ $\left.\delta^{i-2}\right)>0$.

In the following, we explore the relation between decay thresholds and decay order of vertices. If $u, v$ are vertices of a graph $G$ and $\delta_{1}, \delta_{2} \in(0,1), \delta_{1} \neq \delta_{2}$ such that $C_{\delta_{1}}(u)>C_{\delta_{1}}(v)$ but $C_{\delta_{2}}(u)<C_{\delta_{2}}(v)$, then $G$ has a decay threshold in $\left(\delta_{1}, \delta_{2}\right)$ (this follows from the intermediate value theorem used on the function $\left.C_{\delta}(u)-C_{\delta}(v)\right)$. However, in general, the converse is not true. To illustrate this, we will use the following observation on decay centralities of endvertices of cut edges in graphs. $G$ be a graph with a cut edge $u v$, and let $G_{1}, G_{2}$ be two components of $G-u v$; let $D D S_{G_{1}}(u)=\left(1, d_{1}, \ldots, d_{k}\right), D D S_{G_{2}}(v)=$ $\left(1, t_{1}, \ldots, t_{l}\right)$. Without loss of generality, let $k \geq l$ and $\left(1, h_{1}, \ldots, h_{k}\right)$ be a sequence of length $k$ such that, for each $i=1, \ldots, l, h_{i}=t_{i}$ and $h_{j}=0$ for $j=l+1, \ldots, k$. Then

$$
\begin{gathered}
D D S_{G}(u)=\left(1, d_{1}+1, d_{2}+h_{1}, \ldots, d_{i}+h_{i-1}, \ldots, d_{k}+h_{k-1}, h_{k}\right)= \\
\left(1, d_{1}, \ldots, d_{k}, 0\right)+\left(0,1, h_{1}, \ldots, h_{k}\right), \\
D D S_{G}(v)=\left(1, h_{1}+1, h_{2}+d_{1}, \ldots, h_{i}+d_{i-1}, \ldots, h_{k}+d_{k-1}, d_{k}\right)= \\
\left(1, h_{1}, \ldots, h_{k}, 0\right)+\left(0,1, d_{1}, \ldots, d_{k}\right)
\end{gathered}
$$


and

$$
\begin{gathered}
C_{\delta}^{G}(u)-C_{\delta}^{G}(v)=\left[\sum_{i=1}^{k} d_{i} \delta^{i}+1 \cdot \delta+\sum_{i=1}^{k} h_{i} \delta^{i+1}\right]-\left[\sum_{i=1}^{k} h_{i} \delta^{i}+1 \cdot \delta+\sum_{i=1}^{k} d_{i} \delta^{i+1}\right]= \\
C_{\delta}^{G_{1}}(u)-C^{G_{2}} \delta(v)-\delta\left(\sum_{i=1}^{k} d_{i} \delta^{i}-\sum_{i=1}^{k} h_{i} \delta^{i}\right)=(1-\delta)\left(C_{\delta}^{G_{1}}(u)-C^{G_{2}} \delta(v)\right) .
\end{gathered}
$$

Thus, $C_{\delta}^{G}(u)-C_{\delta}^{G}(v)$ has a root $\alpha \in(0,1)$ if and only if $\alpha$ is the root of $C_{\delta}^{G_{1}}(u)-C^{G_{2}} \delta(v)$.

We use this observation to construct connected graphs containing a pair of adjacent vertices showing arbitrary behaviour of their decay centralities. Given non-negative integers $n_{1}, n_{2}$, choose $n_{1}+n_{2}$ rational numbers $q_{1}, \ldots, q_{n_{1}}$, $r_{1}, \ldots, r_{n_{2}} \in(0,1)$ and a positive integer $A$ in such a way that the polynomial $P(x)=A x \prod_{i=1}^{n_{1}}\left(x-q_{i}\right)^{2} \prod_{j=1}^{n_{2}}\left(x-r_{j}\right)=a_{2 n_{1}+n_{2}} x^{2 n_{1}+n_{2}}+\cdots+a_{1}$ has integer coefficients. Next, for $i=1, \ldots, 2 n_{1}+n_{2}$, choose positive integers $b_{i}, c_{i}$ such that $a_{i}=b_{i}-c_{i}$, and construct disjoint connected graphs $G_{1}, G_{2}$ such that there is a vertex $u$ of $G_{1}$ with $D D S_{G_{1}}(u)=\left(1, b_{1}, \ldots, b_{2 n_{1}+n_{2}}\right)$ and a vertex $v$ of $G_{2}$ with $D D S_{G_{2}}(v)=\left(1, c_{1}, \ldots, c_{2 n_{1}+n_{2}}\right)\left(G_{1}\right.$ and $G_{2}$ may be chosen as rooted trees of height $2 n_{1}+n_{2}$ with roots $u$ and $v$ having $b_{i}$ and $c_{i}$ vertices at $i$-th level). Now, let $G$ be a graph obtained from $G_{1}$ and $G_{2}$ by adding a new edge $u v$. It follows that, in $G$, the difference of decay centralities of $u$ and $v$ is equal to $P(\delta)(1-\delta)$, hence $q_{1}, \ldots, q_{n_{1}}, r_{1}, \ldots, r_{n_{2}}$ are decay thresholds of $G$. Observe that, for thresholds $q_{i}, \ldots, q_{n_{1}}$, a small local change of the parameter $\delta$ in their neighbourhoods preserves decay ordering of $u, v$ whereas, for a local change of $\delta$ in neighbourhoods of thresholds $r_{1}, \ldots, r_{n_{2}}$, the order of $u$ and $v$ is always reversed.

Example 1. Consider $q_{1}=\frac{1}{2}, r_{1}=\frac{1}{3}$. Then one can set $P(x)$ being equal to $12 x\left(x-\frac{1}{2}\right)^{2}\left(x-\frac{1}{3}\right)=12 x^{4}-16 x^{3}+7 x^{2}-x$, hence, $a_{4}=12, a_{3}=-16, a_{2}=$ $7, a_{1}=-1$ and we can choose $b_{1}=1, c_{1}=2, b_{2}=8, c_{2}=1, b_{3}=1, c_{3}=$ $17, b_{4}=13, c_{4}=1$. An example of a graph realizing these parameters is on Figure 2; it is easy to see that the decay centralities of vertices $u$ and $v$ are $2 \delta+10 \delta^{2}+2 \delta^{3}+30 \delta^{4}+\delta^{5}$ and $3 \delta+2 \delta^{2}+25 \delta^{3}+2 \delta^{4}+13 \delta^{5}$, respectively, and their difference is $\delta-8 \delta^{2}+23 \delta^{3}-28 \delta^{4}+12 \delta^{5}=\delta(\delta-1)(3 \delta-1)(2 \delta-1)^{2}$ yielding the roots $0,1, \frac{1}{3}$ (single root) and $\frac{1}{2}$ (double root).

From the above examples, one can conclude that the decay centrality, although being well defined for disconnected graphs, may sometimes lead, in these and other graphs, to "unpleasant" issues involving relation between decay order of vertices and decay thresholds. 


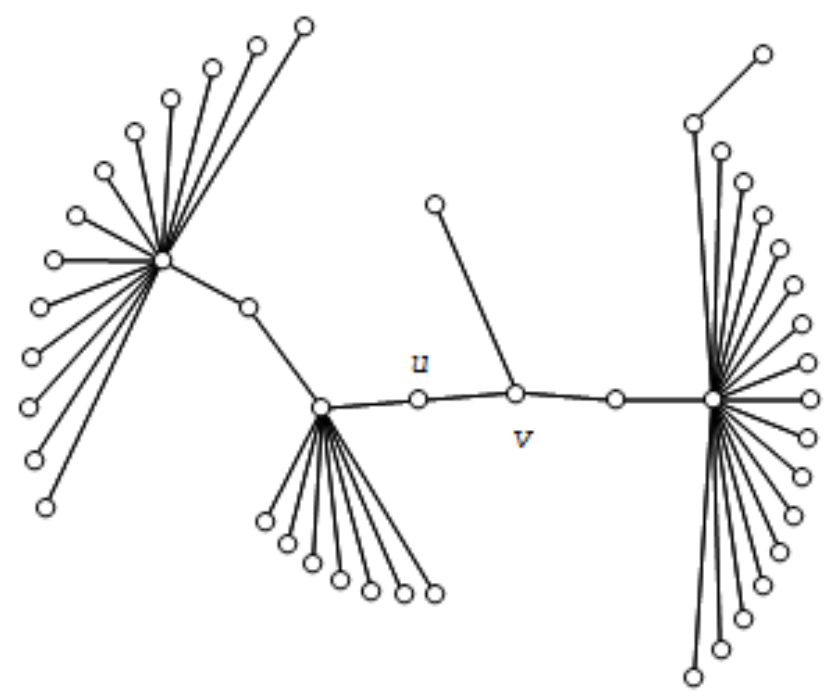

Figure 2: An example of graph with two thresholds of different nature

\section{Decay-stable graphs}

We start our search for decay-stable graphs with several examples of graphs from simple yet nontrivial classes (note that since each vertex-transitive graph is also DDR graph nad therefore decay-stable, we concentrate on classes of nontransitive graphs):

Proposition 2. All paths are decay-stable.

Proof. Let $P_{n}=v_{1} v_{2} \ldots v_{n}$ be an $n$-vertex path. For integer $k, 1 \leq k \leq\left\lfloor\frac{n}{2}\right\rfloor$, we have $C_{\delta}\left(v_{k}\right)=C_{\delta}\left(v_{n-k}\right)=2 \sum_{i=1}^{k-1} \delta^{i}+\sum_{i=k}^{n-k} \delta^{i}=\frac{\delta^{k}+\delta^{n-k+1}-2 \delta}{\delta-1}$. Now, for fixed $n$ and $\delta \in(0,1)$, the function $c(x)=\frac{\delta^{x}+\delta^{n-x+1}-2 \delta}{\delta-1}$ is increasing on $\left(1, \frac{n}{2}\right)$ because $c^{\prime}(x)=\frac{\ln \delta\left(\delta^{x}-\delta^{n-x+1}\right)}{\delta-1}>0$; thus, $1 \leq p<q \leq\left\lfloor\frac{n}{2}\right\rfloor, C_{\delta}\left(v_{p}\right)<C_{\delta}\left(v_{q}\right)$ which proves the claim.

In search for decay-stable trees, we checked, by help of Maple computer algebra system, all trees up to 20 vertices. The numbers of decay-stable trees are given by the following table: 


\begin{tabular}{|c||c|c|c|c|c|c|}
\hline number of vertices & 4 & 5 & 6 & 7 & 8 & 9 \\
\hline $\begin{array}{c}\text { number of decay- } \\
\text { stable trees }\end{array}$ & 2 & 3 & 6 & 9 & 19 & 20 \\
\hline number of all trees & 1 & 3 & 6 & 11 & 23 & 47 \\
\hline \hline number of vertices & 10 & 11 & 12 & 13 & 14 & 15 \\
\hline $\begin{array}{c}\text { number of decay- } \\
\text { stable trees }\end{array}$ & 40 & 49 & 88 & 102 & 191 & 207 \\
\hline number of all trees & 106 & 235 & 551 & 1301 & 3159 & 7741 \\
\hline \hline number of vertices & 16 & 17 & 18 & 19 & 20 & \\
\hline $\begin{array}{c}\text { number of decay- } \\
\text { stable trees }\end{array}$ & 356 & 391 & 678 & 731 & 1265 & \\
\hline number of all trees & 19320 & 48629 & 123867 & 317955 & 823065 & \\
\hline
\end{tabular}

These numbers suggest that, from the asymptotic point of view, the following conjecture is true:

Conjecture 1. Almost every tree is decay-unstable.

It is not easy to find a particular graph constructions producing decaystable graphs from smaller graphs, as the most common graph operations yield, in general, negative results even for decay-stable operands. We illustrate this for several graph products, namely, the Cartesian, tensor, strong and the lexicographic product (the definition and properties of these graph operations can be found in Imrich and Klavžar 2000). For example, the graphs $K_{1,3}, K_{1,3}^{+}$ (that is, $K_{1,3}$ with an extra edge between its nonadjacent vertices), $P_{3}, P_{4}$ and $P_{5}$ are decay-stable, but the Cartesian product $K_{1,5} \square P_{5}$ has unique threshold $\frac{1}{15} \cdot \sqrt[3]{918+30 \sqrt{921}}+\frac{8}{5 \sqrt[3]{918+30 \sqrt{921}}}-\frac{1}{5} \doteq 0.7460547439$, the strong product $K_{1,3} \otimes P_{4}$ and the tensor product $K_{1,3}^{+} \times P_{4}$ have unique threshold $\frac{1}{2}$, and the lexicographic product $P_{5}\left[P_{3}\right]$ has unique threshold $\frac{1}{3}$ (see Figure 3 for detailed visualization of decay curves and their intersections). Nevertheless, for particular factors of these graph products, one can obtain decay stability of the result; we show this by several different examples:

Theorem 1. If $G$ is decay-stable, then, for any positive integer $n, G \square K_{n}$ is also decay-stable.

Proof. Let $u$ be a vertex of $G, D D S(u)=\left(1, d_{1}, \ldots, d_{k}\right)$ where $k=e c_{G}(u)$, and let $[u, v]$ be any vertex of $G \square K_{n}$ which lies in a copy of the factor $K_{n}$ that covers $u$ in a copy of $G$ in the product. Note that for each vertex $[w, z]$ of $G \square K_{n}$ (with $w \in V(G), z \in V\left(K_{n}\right)$ ), the distance of $[u, v],[w, z]$ is equal to $d(u, w)+1$; this implies that $D D S([u, v])=\left(1, d_{1}+n-1, d_{2}+(n-1) d_{1}, \ldots, d_{k}+(n-\right.$ $\left.1) d_{k-1},(n-1) d_{k}\right)$. Hence, for any vertex $[w, z]$ of $G \square K_{n}$ with $D D S_{G}(w)=$ $\left(1, d_{1}^{\prime}, \ldots, d_{l}^{\prime}\right), l=e c_{G}(w)$ (without loss of generality, let $k \leq l$ ), we obtain

$$
C_{\delta}([w, z])-C_{\delta}([u, v])=\sum_{i=0}^{k-1}\left(d_{i+1}^{\prime}+(n-1) d_{i}^{\prime}-\left(d_{i+1}+(n-1) d_{i}\right)\right) \delta^{i+1}+
$$




$$
\begin{gathered}
\left(d_{k+1}^{\prime}+(n-1) d_{k}^{\prime}-(n-1) d_{k}\right) \delta^{k+1}+\sum_{i=k+2}^{l-1}\left(d_{i+1}^{\prime}+(n-1) d_{i}^{\prime}\right) \delta^{i}+(n-1) d_{l}^{\prime} \delta^{l}= \\
\left(C_{\delta}(u)-C_{\delta}(w)\right)(\delta(n-1)+1) .
\end{gathered}
$$

Since $G$ is decay-stable, $C_{\delta}(u)-C_{\delta}(w)$ has no real root in $(0,1)$, thus $C_{\delta}([u, v])-C_{\delta}([w, z])$ has no real root in $(0,1)$ either, which proves the claim.

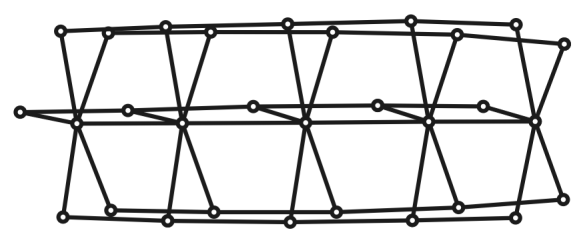

$K_{1,5} \square P_{5}$

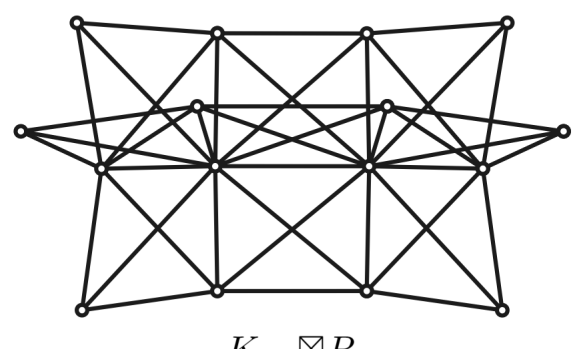

$K_{1,3} \otimes P_{5}$

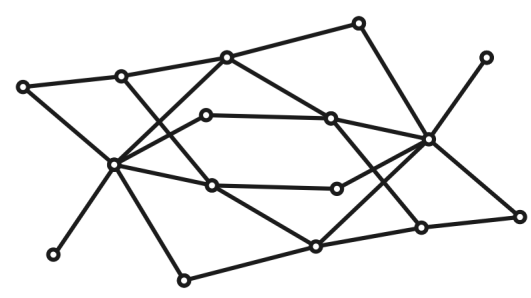

$K_{1,3}^{+} \times P_{4}$

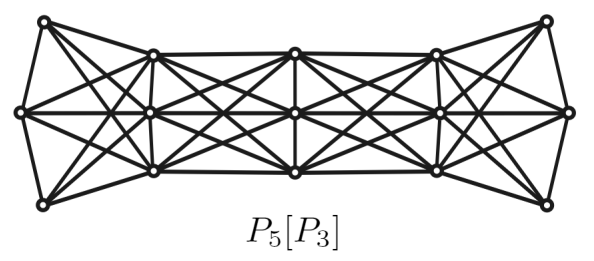

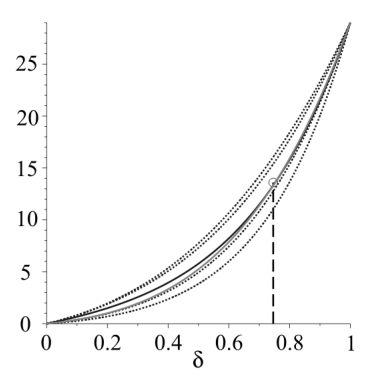
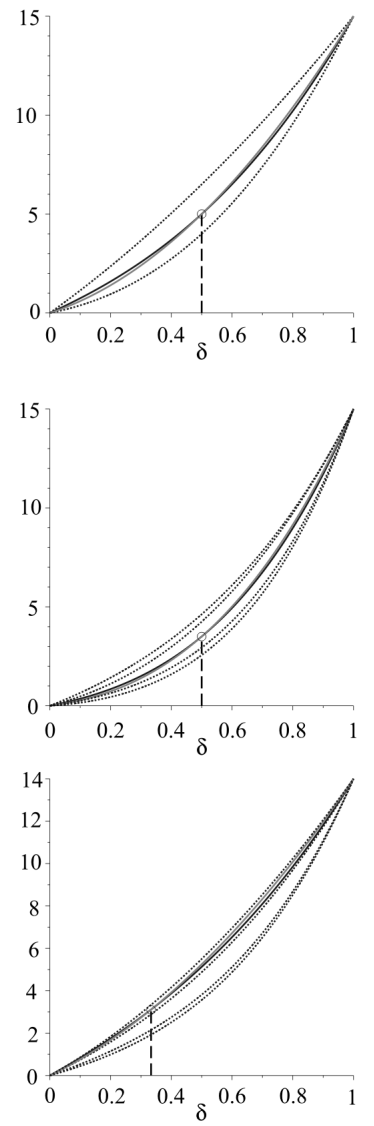

Figure 3: Examples of decay-unstable graph products 
Theorem 2. If $G$ with $\delta(G) \geq 1$ is decay-stable and $H$ is a regular graph, then $G[H]$ is decay-stable.

Proof. Let $H$ be an $r$-regular graph on $s$ vertices, $v$ be a vertex of $H$ and let $u$ be a vertex of $G$ with $e c(u)=k, D D S_{G}(u)=\left(1, d_{1}, \ldots, d_{k}\right)$. Then $D D S_{G[H]}([u, v])=\left(1, d_{1} s+r, d_{2} s+s-r-1, d_{3} s, \ldots, d_{k} s\right)=s \cdot D D S_{G}(u)+$ $(1-s-r, r, s-r-1,0, \ldots, 0)$. Hence, for any two vertices $[u, v],[w, z]$ of $G[H]$, the difference of their decay centralities is

$$
\begin{gathered}
C_{\delta}([u, v])-C_{\delta}([w, z])=s \cdot\left(C_{\delta}(u)-C_{\delta}(w)\right)+(r-r) \delta+(s-r-1-(s-r-1)) \delta^{2}= \\
s \cdot\left(C_{\delta}(u)-C_{\delta}(w)\right) .
\end{gathered}
$$

Again, $G$ is decay-stable, thus, $C_{\delta}(u)-C_{\delta}(w)$ has no real root in $(0,1)$ as well as $C_{\delta}([u, v])-C_{\delta}([w, z])$; this proves the claim.

It seems that also the following is true:

Conjecture 2. For all positive integers $\ell, k_{1}, \ldots, k_{\ell}$, the $\ell$-dimensional grid $P_{k_{1}} \square \ldots \square P_{k_{\ell}}$ is decay-stable.

Conjecture 3. The strong product $P_{k} \otimes P_{l}$ is decay-stable for all positive integers $k, l$.

Before exploring another large set of decay-stable graphs, we prove auxiliary result concerning relative stability of two vertices whose distance profiles do not differ much:

Lemma 2. Let $G$ be a graph and $x, y \in V(G)$. If $D D S(x)$ and $D D S(y)$ differ in exactly two terms, then, for all $\delta \in(0,1), C_{\delta}(x) \neq C_{\delta}(y)$.

Proof. By contradiction. Let $G$ be a graph with two vertices $x, y$ with eccentricities $e_{x}, e_{y}$ such that $D D S(x)=\left(1, x_{1}, \ldots, x_{e_{x}}\right)$ and $D D S(y)=\left(1, y_{1}, \ldots, y_{e_{y}}\right)$ differ exactly in $i$-th and $j$-th terms, $i<j$. Put $k=\max \left\{e_{x}, e_{y}\right\}$. Then $\sum_{l=1}^{k} x_{l}=\sum_{l=1}^{k} y_{l}$, which gives $x_{i}-y_{i}=y_{j}-x_{j}$. Assume that there exists a $\delta \in(0,1)$ such that $C_{\delta}(x)=C_{\delta}(y)$. This implies that $x_{i} \delta^{i}+x_{j} \delta^{j}=y_{i} \delta^{i}+y_{j} \delta^{j}$, hence $x_{i}-y_{i}=\left(y_{j}-x_{j}\right) \delta^{j-i}$. Thus $\delta^{j-i}=1$, a contradiction.

Since all pairs of vertices of graphs of diameter 2 satisfy the above Lemma, we obtain the following

Corollary 1. All graphs of diameter 2 are decay-stable.

This also shows that - in probabilistic sense involving the concept of random graphs - almost every graph is decay-stable, as well as all joins of graphs. Furthermore, each strongly regular graph (that is, a regular graph with the property that every two adjacent vertices have $\lambda$ common neighbours and evey 
two nonadjacent vertices have $\mu$ common neighbours) has diameter 2 , thus, it is decay-stable. By Phelps (1979), every finite group $A$ can serve as an automorphism group of some strongly regular graph, and hence of a decay-stable graph.

Corollary 2. All regular graphs of diameter 3 are decay-stable.

Note that there are nonregular graphs of diameter 3 which are not decaystable, for example, the graph on Figure 4. Also, there are regular graphs of diameter at least 4 which are not decay stable - for example, the cubic graph on Figure 5 has decay threshold $\frac{1}{2}$. Hence, the assumptions of both above corollaries are best possible.

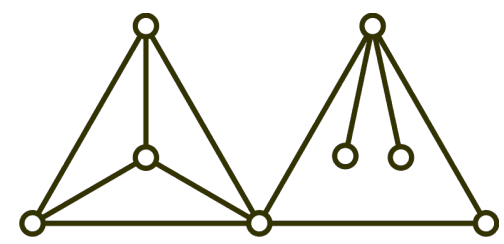

Figure 4: An example of decay-unstable graph of diameter 3

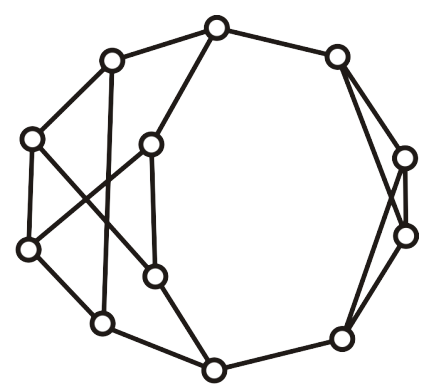

Figure 5: An example of decay-unstable regular graph of diameter 4

On the other hand, it seems that most of real-world networks are decayunstable. We have checked this property for several well-known networks (the data files are found at http://www-personal.umich.edu/ mejn/netdata/): the network of Zachary karate club (see Zachary 1977; describing friendships between 34 members of a karate club at a US university in the 1970s), the dolphin social network (see Lusseau et al. 2003) of frequent associations between 62 dolphins in a community living off Doubtful Sound, New Zealand, and the Padgett's network (see Padgett and Ansell 1993) of marriages between Florentine medieval families. These networks have high numbers of decay thresholds using Maple computer algebra system, we have determined that the Padgett's network has seven thresholds while Zachary karate club network and the dolphin social network have 35 and 372 (!) thresholds, respectively. It would be interesting to compare these numbers with statistical characteristics of threshold numbers of random graphs with main parameters (number of vertices and edges, diameter) being the same as for real-world networks. 


\section{References}

Reinhard Diestel. Graph theory (Graduate texts in mathematics). Springer Heidelberg, 2005.

Ulrik Brandes and Thomas Erlebach. Network analysis: methodological foundations, volume 3418. Springer Science \& Business Media, 2005.

Vito Latora and Massimo Marchiori. Efficient behavior of small-world networks. Physical review letters, 87(19):198701, 2001.

M. Knor and T Madaras. On farness-and reciprocally-selfcentric antisymmetric graphs. Congr. Numer, 171:173-178, 2004.

Matthew O. Jackson et al. Social and economic networks, volume 3. Princeton University Press Princeton, 2008.

Matthew O. Jackson and Asher Wolinsky. A strategic model of social and economic networks. Journal of economic theory, 71(1):44-74, 1996.

Chavdar Dangalchev. Residual closeness and generalized closeness. International Journal of Foundations of Computer Science, 22(08):1939-1948, 2011.

Rong Yang and Leyla Zhuhadar. Extensions of closeness centrality? In Proceedings of the 49th Annual Southeast Regional Conference, pages 304-305. ACM, 2011.

Gary S. Bloom, John W. Kennedy, and Louis V. Quintas. Some problems concerning distance and path degree sequences. In Graph Theory, pages 179190. Springer, 1983.

Wilfried Imrich and Sandi Klavžar. Product graphs. Wiley, 2000.

KT Phelps. Latin square graphs and their automorphism groups. Ars Combin, 7:273-299, 1979

Wayne W Zachary. An information flow model for conflict and fission in small groups. Journal of anthropological research, pages 452-473, 1977.

David Lusseau, Karsten Schneider, Oliver J. Boisseau, Patti Haase, Elisabeth Slooten, and Steve M. Dawson. The bottlenose dolphin community of doubtful sound features a large proportion of long-lasting associations. Behavioral Ecology and Sociobiology, 54(4):396-405, 2003.

John F. Padgett and Christopher K. Ansell. Robust action and the rise of the medici, 1400-1434. American journal of sociology, pages 1259-1319, 1993. 